\title{
Prenatal Diagnosis Nomograms: A Novel Tool to Predict Fetal Chromosomal Abnormalities in High-Risk Patients
}

\author{
Yangzi Zhou' \\ Zixuan Song' \\ Lu Sun ${ }^{1,2}$ \\ Yuting Wang' \\ Xiting Lin' \\ Dandan Zhang $\mathbb{I D}^{\prime}$ \\ 'Shengjing Hospital of China Medical \\ University, Department of Obstetrics and \\ Gynecology, Shenyang, People's Republic \\ of China; ${ }^{2}$ Shengjing Hospital of China \\ Medical University, Department of \\ Clinical Genetics, Shenyang, People's \\ Republic of China
}

Correspondence: Dandan Zhang Shengjing Hospital of China Medical University, Department of Obstetrics and Gynecology, Shenyang, People's Republic of China

Email zhangdd@sj-hospital.org
Background: Amniocentesis is an invasive prenatal diagnostic technique that can provide genetic information of fetus for pregnant women and give them a choice. A straightforward predictive tool can show pregnant women the need for amniocentesis prior to the procedure. Methods: The information of patients who underwent amniocentesis from 2014 to 2019 at the Obstetrics Clinic, Shengjing Hospital of China Medical University was extracted, and important independent prognostic factors were determined by univariate and multivariate logistic regression analysis to construct nomograms with total abnormalities (TA) and chromosome number abnormalities (CNA).

Results: A total of 19,683 patients undergoing amniocentesis were included in this study. Among 1761 patients with abnormal results, 917 had abnormal chromosome numbers, 439 had abnormal chromosome structures, and 405 had polymorphic results. Nomograms of TA and CNA were created using data such as age, nuchal translucency value, ultrasound results, Oscar's testing and/or non-invasive prenatal testing abnormalities, parental chromosomes, and information whether they were twins. The nomogram has good predictive power and clinical practicality through the analysis of area under curve and decision curve analysis. Internal verification was performed for nomograms of TA and CNA, suggesting that the nomogram's predicted probability and actual probability of the two are consistent.

Conclusion: The nomogram constructed is a good predictor of TA and CNA, which can be used in clinical practice to screen high-risk patients of chromosomal abnormalities.

Keywords: nomogram, amniocentesis, chromosome abnormal, prenatal diagnosis

\section{Introduction}

At present, the prevalence of chromosomal abnormality in early pregnancy abortions is clinically confirmed in more than $50 \%$ cases, ${ }^{1}$ and fetal chromosomal aneuploidy accounts for $6-11 \%$ of stillbirths and neonatal deaths. ${ }^{2}$ Newborns with chromosomal abnormalities who survived accounted for $0.65 \%$ of all newborns, and chromosomal structural abnormalities that ultimately affected fertility accounted for $0.2 \%$ of newborns. ${ }^{3}$ Therefore, prenatal screening and diagnosis of chromosomal abnormalities has important economic significance and social benefits for improving pregnancy outcomes and giving to the pregnant woman a "sure" diagnosis to decide the outcome of pregnancy.

Prenatal screening is mainly divided into two types: one is fetal morphological level examination, using high-definition ultrasound to check whether the biometry and the anatomy are normal; and the other using maternal blood, urine, and other 
special tests, such as alpha-fetoprotein test (AFP) and, unconjugated estriol test (UE3) to predict fetal neural tube defects (NTDs) or trisomy 21 on the basis of metabolites and enzymology. Prenatal diagnosis can be improved with the positive results or high-risk factors identified during prenatal screening. Invasive prenatal diagnosis is used to diagnose fetal diseases through direct access to maternal amniotic fluid, fetal blood, and tissues. Amniocentesis is one of the most common methods for prenatal diagnosis, and it provides an effective and reliable way to obtain fetal genetic material. The appropriate puncture time is during 16-24 weeks of gestation, when the procedure is easier to perform. The amniotic fluid can be pumped to obtain $20 \mathrm{~mL}$ of the fluid so that there are enough living cells in the amniotic fluid for culture. A multi-center study has confirmed that ultrasonic-guided amniocentesis is now one of the definitive diagnostic methods, with over $99 \%$ accuracy in the diagnosis of chromosomal disorders. ${ }^{4}$

Although amniocentesis is a relatively safe prenatal diagnostic technique, studies have shown that amniocentesis has a miscarriage rate of $1 / 300$ to $1 / 500$, and the miscarriage rate may be even lower when performed by experienced medical professionals. Leakage of amniotic fluid from the puncture hole may occur in about $1-2 \%$ of cases, and the outcome is usually normal. A small leakage of amniotic fluid usually stops within a week naturally, and perinatal survival is more than $90 \%$ in patients with amniotic fluid leakage in the second trimester after amniocentesis. ${ }^{5}$ Fetal injury is also thought to be a complication in the twentieth century, the injury rate is $1-3 \%{ }^{6}$ However, in continuous ultrasoundguided amniocentesis, the injury caused by needling the fetus rarely occurs. It has been reported that long-term follow-up of live births of pregnant women who underwent amniocentesis did not increase the incidence of disability as compared with controls who did not undergo amniocentesis. ${ }^{5}$ There is also a possibility of intrauterine infections, although they can be avoided by strict aseptic practices. Some infections, such as hepatitis $\mathrm{B} \& \mathrm{C}$, and human immunodeficiency virus, have also been reported to be associated with amniocentesis, which increases the risk of mother-to-child transmission from invasive procedures. ${ }^{7,8}$

Because the diagnosis of chromosomal abnormality through amniotic fluid is limited by the time of pregnancy, the optimal gestational age for pregnant women is 16-24 weeks. ${ }^{9}$ However, in 16 to 20 weeks, pregnant women can feel the fetal movement clearly and emotionally connect with the fetus. During that phase, they and their families are waiting anxiously and nervously for the results for 2-3 weeks, which is quite a difficult process. If a fetal chromosomal abnormality is found, the pregnancy may have to be terminated, and the physical injury, mental torture, and risk of surgical procedure caused by mid-pregnancy induction is significantly increased. Chromosomal culture fails was also a rare complication, with an incidence of $0.5 \%$ during 1990 1994 and declining with the development of technology. ${ }^{10}$ However, if the chromosomal culture fails, it may become necessary to choose umbilical cord blood puncture, with the increase in gestational age beyond the time of amniotic fluid puncture, which will further increase the mental pressure of pregnant women and their families. Therefore, although amniocentesis is regarded as the gold standard for the diagnosis of fetal chromosomal diseases, the invasive procedures and associated complications may cause unnecessary complications in pregnant women. International guidelines like the one published by the SIEOG, ${ }^{11} \mathrm{ACOG}^{12}$ or $\mathrm{RCOG}^{13}$ are really clear in explaining when to perform amniocentesis and risk table determining the probability of positive amniocenteses, and gynecology and geneticist must inform the pregnant of the risk and benefits of the procedure, but amniocentesis is requested only by the woman and only the woman will decide what to do. A straightforward predictive tool and a visualized risk-scoring system are contributed to judgments for pregnant women.

A nomogram which is developed based on logistic regression analysis with multiple factors provides accurate prediction in various situations. It represents as a graphical presentation of a prediction model which is widely used to predict the incidence and prognosis of diseases, and in recent years, obstetricians and gynecologists have started using them. ${ }^{14,15}$ To the best of our knowledge, there is no nomogram for predicting abnormal amniocentesis outcomes. In this study, a nomogram of amniocentesis results was established by assessing the factors associated with abnormal results of amniocentesis and was based on the outpatient visits data of the patients undergoing amniocentesis at our center. The purpose of this study was to combine the risk factors associated with positive amniocentesis results into a prediction nomogram based on the data from a single center, large-population institution.

\section{Materials and Methods \\ Study Population}

We conducted a retrospective study of pregnant women who underwent amniocentesis from January 2014 to 
December 2019 at Shengjing Hospital, China Medical University. All the cases underwent consultation during 18 to 25 weeks of gestation at the Clinic of Genetic Counseling, Maternal Fetal Medicine, and General Obstetrics, and accorded with the indications of prenatal diagnosis. The criteria for amniocentesis was as follows: 1) maternal age of the pregnant woman $\geq 35$ years old at delivery; 2) pregnant woman with a history of conceiving children with chromosomal abnormalities; 3 ) one of the spouses has an abnormal chromosomal structure; 4) abnormal maternal serum screening test, defined as a risk $\geq 1$ / 270 for trisomy- 21 and a risk $\geq 1 / 100$ for trisomy-18 in triple in the second trimester Oscar Test; 5) non-invasive prenatal testing (NIPT) showed that absolute z-score $>3$ and L score $>1 ; 6$ ) history of exposure to drugs or poisons with embryotoxicity, fetotoxicity or development toxicant during pregnancy; 7) ultrasound examination revealed fetal abnormalities; 8) the patient demands it strongly for personal reasons. All clinical data for these cases were obtained from the health information system of our institution. Patients with incomplete clinical data were excluded from the study cohort.

Fetal ultrasound abnormalities included circulatory system, respiratory system, urinary system, digestive system and nervous system abnormalities. In addition, fetal appendage abnormalities screened for included single umbilical arteries, amniotic fluid volume disorder, abnormal masses of the placenta or umbilical cord, and omphalocele defects. Appearance and morphological abnormalities screened for included cleft lips and palates, short or missing nasal bones, dysplasia of the limbs, FGR, neck lymphangiomas, diaphragmatic hernias, and hermaphroditism.

All included patients had signed informed consent. This study was approved by the Ethical Review Committee of Shengjing Hospital, China Medical University, and conformed to the principles outlined in the Declaration of Helsinki (World Medical Association Declaration of Helsinki).

\section{Chromosomal Karyotype Analysis}

After centrifugation, two $15-\mathrm{mL}$ bottles of amniotic fluid were inoculated into a culture bottle under aseptic operation conditions. The cells were cultured in an incubator with 5\% $\mathrm{CO} 2$ at $37{ }^{\circ} \mathrm{C}$ for $10-14$ days. When the culture grew well, colchicine was added to the cells and then the cells were recycled. The conventional method was used to make the slides and perform G-banding. At least 30 fission phases were counted and five karyotypes were analyzed under the microscope in each case. If there was a suspected abnormal karyotype or chimeric type, the number of fission phases was to exceed 50. Our chromosome naming method is described in the International System for Human Cytogenetic Nomenclature (2013) (ISCN). ${ }^{16}$

\section{Data Collection}

Data on demographics and clinical characteristics of the patients were extracted, including age, nuchal translucency (NT) value, ultrasound results, history of adverse pregnancy, Down syndrome screening and/or noninvasive DNA test results, parental chromosomes, a history of exposure to harmful substances, data on whether fetal were twins, and whether the parents had familial genetic disorders. Amniocentesis results were defined as either normal or abnormal. Abnormal results include chromosome number abnormality, chromosome structure abnormality, and polymorphism.

\section{Statistical Analysis}

R-version 3.6.3 (R Foundation for Statistical Computing, Vienna, Austria, http://www.r-project.org) was used to analyze all data in the R-Studio environment. $\mathrm{p}<0.05$ was considered to be statistically significant. Univariate and multivariate logistic regression analyses were performed using clinical data to assess factors associated with abnormal amniotic fluid puncture results. Odds ratios (ORs) and 95\% confidence intervals (CIs) were calculated. Abnormal amniocentesis results were predicted by establishing nomograms of patients who underwent amniocentesis according to the relevant risk factors. The calibration of nomograms was evaluated by bootstrapping (1000 re-samplings) to generate a calibration chart. Nomograms were evaluated by studying the area under the receiver operating characteristic (ROC) curve (AUC). ${ }^{17}$ Internal validation through bootstrapping (1000 RES amplification), concordance statistic $\left(\mathrm{C}\right.$-statistic) ${ }^{18}$ and Brier score ${ }^{19}$ were compared between the original model and the validated model. The clinical effects of the nomogram were assessed by the decision curve analysis (DCA), ${ }^{20}$ and the net benefit at each risk threshold probability was calculated.

\section{Results}

\section{Patient Characteristics}

From 2014 to 2019, a total of 20,103 patients provided consent for amniocentesis at Shengjing Hospital of China 
Medical University, and out of them, 19,683 patients matched the inclusion criteria for this study. Among 1761 patients with abnormal results, 917 had abnormal chromosome numbers, 439 had abnormal chromosome structures, and 405 had polymorphic results. The average age of the patients was $31.54 \pm 5.33$ years, and the average gestational age was 22.54 \pm 3.01 months. Among them, 18,729 cases were successful during first-time puncture, and the success rate was $93.17 \%$. The majority of patients with abnormal results were from 30-40 years old (54\%). The specific characteristics of the patients are shown in Table 1.

\section{Risk Factor Analysis of Abnormal Results}

After examination and transformation of variables to fit the logistic regression model, variables were selected using the backward stepwise selection method $(p<0.05)$. The univariate and multivariate logistic regression of total abnormalities (TA) is shown in Table 2, and of the chromosomal number abnormality (CNA) is shown in Table 3. Univariate and multivariate analyses showed that both the TA and CNA were associated with older age, higher NT values, ultrasound abnormalities, Oscar's Test and/or NIPT, parental chromosomal abnormalities, and presence of twins.

\section{Nomogram Construction}

A nomogram of TA and CNA was constructed on the basis of the important variables of multivariate logistic regression, including age, NT value, ultrasound results, Oscar's Test and/or NIPT, parental chromosome results, and whether or not the fetal were twins (Figure 1).

\section{Performance of Nomograms}

The ROC curve of nomograms used to evaluate TA and CNA are shown in Figure 2. The area under the curve (AUC) of nomograms constructed by using the variables selected in multiple logistic regression were higher than $60 \%$ for both, indicating that the nomograms could predict the TA and CNA well. In addition, both DCA (Figure 3) suggest that nomograms have good clinical benefits. Internal verification was performed for nomograms of TA and CNA. The calibration curves of both nomograms were close to the $45^{\circ}$ line, suggesting that the predicted probability of both nomograms was consistent with the actual probability (Figure 4). The C-statistic and Brier score before and after internal verification are shown in Table 4. The internal verification of the two groups of nomograms indicates that the predicted value has good consistency.

\section{Discussion}

In recent years, due to a lot of attention being given to prenatal diagnosis nationally, awareness among people regarding prenatal and postnatal care, environmental pollution, gradually increasing age of pregnant women, and the rapid development of the use of ultrasound technology during pregnancy, the number of pregnant women included in the category of prenatal diagnosis has also increased rapidly. In the population requiring prenatal diagnosis, most pregnant women need to use invasive methods for prenatal diagnosis to give a "sure" diagnosis to woman and to let her decide the outcome of the pregnancy, ${ }^{21,22}$ in addition to the screening for obvious multiple fetal malformations or structural changes that can be diagnosed by ultrasound or MRI. However, amniocentesis may have a series of complications, such as amniotic fluid leakage, premature rupture of membranes, direct or indirect fetal injury, infection, and abortion, and the mother may also be complicated by chorioamnionitis. Therefore, pregnant women still show concerns when they are faced with the choice of prenatal diagnostic techniques. Therefore, we constructed a nomogram for positive results in amniocentesis, which can provide evidence for necessary prenatal diagnosis.

Pergment's study ${ }^{23}$ indicated that the most common prenatal diagnosis indicator was advanced age, followed by positive serum screening and abnormal ultrasonic indicators. Among them, women with advanced age refer to pregnant women older than 35 years at the time of delivery. Due to the ovarian function degeneration and gradual aging of eggs, the probability of abnormal chromosome meiosis gradually increases. According to some reports, pregnant women with advanced age, especially those over 40 years old, can skip serum screening and can directly undergo prenatal diagnosis. ${ }^{24,25}$ A study that divided pregnant women into groups: 35-37 years old, 38-40 years old, and $\geq 40$ years of age for comparison, found that fetal chromosomal abnormality detection rate increased significantly in the $\geq 40$ years old group. ${ }^{26}$ NIPT uses fetal free DNA from maternal plasma for next-generation sequencing for prenatal screening of aneuploidy risk assessment, which can detect $99 \%$ of trisomy $21,98 \%$ of trisomy 18 , and $99 \%$ of trisomy $13 .{ }^{27}$ However, the accuracy of this technique in the examination of sex chromosome aneuploidy varies greatly, ${ }^{28}$ and NIPT does not detect structural 
Table I Characteristics of the High-Risk Patients and Their Amniocentesis Results

\begin{tabular}{|c|c|c|c|c|c|}
\hline Characteristics & $\begin{array}{l}\text { Normal } \\
N=\mid 7922\end{array}$ & $\begin{array}{l}\text { Total } \\
\text { Abnormalities } \\
\mathrm{N}=|76|\end{array}$ & $\begin{array}{l}\text { Chromosome } \\
\text { Number Abnormality } \\
\mathrm{N}=917\end{array}$ & $\begin{array}{l}\text { Chromosomal } \\
\text { Structural } \\
\text { Abnormality } N=439\end{array}$ & $\begin{array}{l}\text { Polymorphic } \\
\text { Abnormality } \\
\mathrm{N}=405\end{array}$ \\
\hline \multicolumn{6}{|l|}{ Age } \\
\hline$<20$ & 192 (1.1\%) & $13(0.7 \%)$ & $6(0.7 \%)$ & $5(1.1 \%)$ & $2(0.5 \%)$ \\
\hline $21-30$ & $8200(46 \%)$ & 695 (39\%) & $329(36 \%)$ & 197 (45\%) & $169(42 \%)$ \\
\hline $31-35$ & $4045(23 \%)$ & 402 (23\%) & 192 (21\%) & $88(20 \%)$ & $97(24 \%)$ \\
\hline $36-40$ & 4635 (26\%) & $54 I(3 I \%)$ & 317 (35\%) & $130(30 \%)$ & 119 (29\%) \\
\hline$>40$ & $850(4.7 \%)$ & 110 (6.2\%) & 73 (8.0\%) & $19(4.3 \%)$ & 18 (4.4\%) \\
\hline \multicolumn{6}{|l|}{ NT (mm) } \\
\hline$<2.5$ & $17,217(96 \%)$ & $1642(93 \%)$ & 842 (92\%) & $4 \mid 3(94 \%)$ & 387 (96\%) \\
\hline $2.5-4.0$ & 493 (2.8\%) & 69 (3.9\%) & $37(4.0 \%)$ & $20(4.6 \%)$ & $12(3.0 \%)$ \\
\hline $4.0-6.0$ & $133(0.7 \%)$ & $31(1.8 \%)$ & $23(2.5 \%)$ & 5 (I.1\%) & $3(0.7 \%)$ \\
\hline$>6.0$ & $79(0.4 \%)$ & $19(1.1 \%)$ & 15 (1.6\%) & I (0.2\%) & \\
\hline \multicolumn{6}{|l|}{ Ultrasound results } \\
\hline Normal & I3,883 (77\%) & 1463 (83\%) & 762 (83\%) & 364 (83\%) & 337 (83\%) \\
\hline Single anomaly & $3480(19 \%)$ & $234(13 \%)$ & 115 (13\%) & 64 (15\%) & $55(14 \%)$ \\
\hline Composite anomaly & $559(3.1 \%)$ & $64(3.6 \%)$ & 40 (4.4\%) & II (2.5\%) & $13(3.2 \%)$ \\
\hline \multicolumn{6}{|l|}{ Maternal history } \\
\hline Normal & 16,397 (91.5\%) & I 647 (94.5\%) & 880 (96\%) & 401 (91\%) & 366 (90\%) \\
\hline Abnormal & 1525 (8.5\%) & 114 (6.5\%) & $37(4.0 \%)$ & 38 (8.7\%) & $39(9.6 \%)$ \\
\hline \multicolumn{6}{|c|}{ Oscar's testing and/or NIPT } \\
\hline Normal & $3572(20 \%)$ & $223(13 \%)$ & 98 (11\%) & 65 (15\%) & $60(15 \%)$ \\
\hline High risk of trisomy 13 & $53(0.3 \%)$ & $14(0.8 \%)$ & $9(1.0 \%)$ & $4(0.9 \%)$ & I (0.2\%) \\
\hline High risk of trisomy 18 & $482(2.7 \%)$ & $89(5.1 \%)$ & $68(7.4 \%)$ & $12(2.7 \%)$ & $9(2.2 \%)$ \\
\hline High risk of trisomy 21 & $5916(33 \%)$ & 587 (33\%) & $348(38 \%)$ & $96(22 \%)$ & 143 (35\%) \\
\hline $\begin{array}{l}\text { Sex chromosome } \\
\text { abnormality }\end{array}$ & $315(1.8 \%)$ & $102(5.8 \%)$ & 77 (8.4\%) & $12(2.7 \%)$ & $13(3.2 \%)$ \\
\hline Other anomaly & 155 (0.9\%) & $21(1.2 \%)$ & $8(0.9 \%)$ & $8(1.8 \%)$ & $5(1.2 \%)$ \\
\hline Composite anomaly & $15(0.08 \%)$ & $3(0.2 \%)$ & $2(0.2 \%)$ & I (0.2\%) & $0(0 \%)$ \\
\hline Neither was tested & $74 \mid 4(41 \%)$ & $722(41 \%)$ & 07 (33\%) & $24 \mid(55 \%)$ & $174(43 \%)$ \\
\hline \multicolumn{6}{|l|}{ Parental chromosomes } \\
\hline Both are normal & $|7,42|(97.2 \%)$ & $1626(92.3 \%)$ & 896 (98\%) & 336 (77\%) & 394 (97\%) \\
\hline $\begin{array}{l}\text { One or both of them are } \\
\text { abnormal }\end{array}$ & $50 \mathrm{I}(2.8 \%)$ & 135 (7.7\%) & $21(2.3 \%)$ & $103(23 \%)$ & II (2.7\%) \\
\hline \multicolumn{6}{|c|}{ History of exposure to hazardous substances } \\
\hline No & 17,627 (98.4\%) & I745 (99.1\%) & 911 (99\%) & 434 (99\%) & 400 (99\%) \\
\hline Yes & $295(1.6 \%)$ & $16(0.9 \%)$ & $6(0.7 \%)$ & $5(1.1 \%)$ & $5(1.2 \%)$ \\
\hline \multicolumn{6}{|l|}{ Twins } \\
\hline No & $|7,4| 7(97.2 \%)$ & 1688 (95.9\%) & 876 (96\%) & 419 (95\%) & $393(97 \%)$ \\
\hline Yes & $505(2.8 \%)$ & 73 (4.1\%) & $4 \mathrm{I}(4.5 \%)$ & $20(4.6 \%)$ & $12(3.0 \%)$ \\
\hline \multicolumn{6}{|l|}{ Familial disease } \\
\hline No & $17,866(99.7 \%)$ & 1756 (99.7\%) & $916(100 \%)$ & 437 (100\%) & 403 (100\%) \\
\hline Yes & $56(0.3 \%)$ & $5(0.3 \%)$ & I (0.1\%) & $2(0.5 \%)$ & $2(0.5 \%)$ \\
\hline
\end{tabular}

Abbreviations: NT, nuchal translucency; NIPT, non-invasive prenatal testing. 
Table 2 The Univariate and Multivariate Logistic Regression Analysis of the Total Abnormalities

\begin{tabular}{|c|c|c|c|c|c|c|}
\hline \multirow[t]{2}{*}{ Characteristic } & \multicolumn{3}{|c|}{ Univariate Logistic Regression } & \multicolumn{3}{|c|}{ Multivariate Logistic Regression } \\
\hline & OR & $95 \% \mathrm{Cl}$ & p-value & OR & $95 \% \mathrm{Cl}$ & p-value \\
\hline \multicolumn{7}{|l|}{ Age } \\
\hline$\leq 20$ & Ref & & & Ref & & \\
\hline $21-30$ & 1.25 & $0.74,2.32$ & 0.438 & 1.22 & $0.72,2.26$ & 0.501 \\
\hline $31-34$ & 1.47 & $0.86,2.73$ & 0.188 & 1.42 & $0.83,2.64$ & 0.236 \\
\hline $36-40$ & 1.72 & $1.02,3.20$ & 0.061 & 2.42 & $\mathrm{I} .4 \mathrm{I}, 4.53$ & $0.003 *$ \\
\hline$>40$ & 1.91 & $1.09,3.63$ & $0.033^{*}$ & 3.02 & $1.70,5.79$ & $<0.001 *$ \\
\hline \multicolumn{7}{|l|}{ NT (mm) } \\
\hline$\leq 2.5$ & Ref & & & Ref & & \\
\hline $2.5-4.0$ & 1.47 & $1.13,1.88$ & $0.003 *$ & 2.59 & $1.93,3.42$ & $<0.001 *$ \\
\hline $4.0-6.0$ & 2.44 & $1.62,3.57$ & $<0.00 I^{*}$ & 4.25 & $2.76,6.38$ & $<0.001 *$ \\
\hline$>6.0$ & 2.52 & $1.48,4.08$ & $<0.00 I^{*}$ & 4.36 & $2.5 \mathrm{I}, 7.24$ & $<0.001 *$ \\
\hline \multicolumn{7}{|l|}{ Ultrasound results } \\
\hline Normal & Ref & & & Ref & & \\
\hline Single anomaly & 0.64 & $0.55,0.73$ & $<0.00 I^{*}$ & 1.26 & $0.97,1.62$ & 0.0839 \\
\hline Composite anomaly & 1.09 & $0.83,1.40$ & 0.539 & 1.80 & $1.29,2.48$ & $<0.001 *$ \\
\hline \multicolumn{7}{|l|}{ Maternal history } \\
\hline Normal & Ref & & & Ref & & \\
\hline Abnormal & 0.74 & $0.6 \mathrm{I}, 0.90$ & $0.003 *$ & 1.25 & $1.00,1.55$ & 0.051 \\
\hline \multicolumn{7}{|l|}{ Oscar's testing and/or NIPT } \\
\hline Normal & Ref & & & Ref & & \\
\hline High risk of trisomy 13 & 4.23 & $2.23,7.53$ & $<0.00 I^{*}$ & 4.35 & $2.19,8.19$ & $<0.001 *$ \\
\hline High risk of trisomy 18 & 2.96 & $2.26,3.84$ & $<0.00 I^{*}$ & 3.65 & $2.57,5.17$ & $<0.001 *$ \\
\hline High risk of trisomy 21 & 1.59 & $1.36,1.87$ & $<0.00 I^{*}$ & 2.08 & I.57, 2.76 & $<0.001 *$ \\
\hline Sex chromosome abnormality & 5.19 & $3.98,6.72$ & $<0.00 I^{*}$ & 5.67 & $3.98,8.05$ & $<0.001 *$ \\
\hline Other anomaly & 2.17 & I.3I, 3.42 & $0.001^{*}$ & 2.44 & $1.41,4.08$ & $<0.001 *$ \\
\hline Composite anomaly & 3.20 & $0.74,9.80$ & 0.067 & 4.58 & I.04, I 4.4 & $0.019 *$ \\
\hline Neither was tested & 1.56 & $1.34,1.83$ & $<0.001 *$ & 0.93 & $0.71,1.23$ & 0.631 \\
\hline \multicolumn{7}{|l|}{ Parental chromosomes } \\
\hline Both are normal & Ref & & & Ref & & \\
\hline One or both of them are abnormal & 2.89 & $2.36,3.50$ & $<0.00 I^{*}$ & 5.11 & $4.06,6.40$ & $<0.00 I^{*}$ \\
\hline \multicolumn{7}{|c|}{ History of exposure to hazardous substances } \\
\hline No & Ref & & & Ref & & \\
\hline Yes & 0.55 & $0.32,0.88$ & $0.020^{*}$ & 1.03 & $0.59,1.67$ & 0.916 \\
\hline \multicolumn{7}{|l|}{ Twins } \\
\hline No & Ref & & & Ref & & \\
\hline Yes & 1.49 & $1.15,1.90$ & $0.002 *$ & 1.76 & I.3I, 2.34 & $<0.001 *$ \\
\hline \multicolumn{7}{|l|}{ Familial disease } \\
\hline No & Ref & & & - & - & - \\
\hline Yes & 0.91 & $0.32,2.06$ & 0.8 & - & - & - \\
\hline
\end{tabular}

Note: *Means $\mathrm{P}<0.05$.

Abbreviations: OR, odds ratio; $\mathrm{Cl}$, confidence interval; NT, nuchal translucency; NIPT, non-invasive prenatal testing; Ref, reference. 
Table 3 The Univariate and Multivariate Logistic Regression Analysis of Chromosome Number Abnormality

\begin{tabular}{|c|c|c|c|c|c|c|}
\hline \multirow[t]{2}{*}{ Characteristic } & \multicolumn{3}{|c|}{ Univariate Logistic Regression } & \multicolumn{3}{|c|}{ Multivariate Logistic Regression } \\
\hline & OR & $95 \% \mathrm{Cl}$ & p-value & OR & $95 \% \mathrm{Cl}$ & p-value \\
\hline \multicolumn{7}{|l|}{ Age } \\
\hline$\leq 20$ & Ref & & & Ref & & \\
\hline $21-30$ & 1.28 & $0.62,3.28$ & 0.550 & 1.34 & $0.64,3.45$ & 0.4835 \\
\hline $31-34$ & 1.52 & $0.73,3.90$ & 0.321 & 1.64 & $0.78,4.23$ & 0.2433 \\
\hline $35-40$ & 2.19 & $1.05,5.59$ & 0.061 & 4.24 & $2.01,10.9$ & $<0.001 *$ \\
\hline$>40$ & 2.75 & $1.28,7.16$ & $0.019 *$ & 6.08 & $2.78,16.0$ & $<0.001 *$ \\
\hline \multicolumn{7}{|l|}{ NT (mm) } \\
\hline$\leq 2.5$ & Ref & & & Ref & & \\
\hline $2.5-4.0$ & 1.53 & $1.07,2.13$ & $0.014 *$ & 3.51 & $2.37,5.06$ & $<0.001 *$ \\
\hline $4.0-6.0$ & 3.54 & $2.20,5.42$ & $<0.001 *$ & 7.99 & $4.80,12.8$ & $<0.001 *$ \\
\hline$>6.0$ & 3.88 & $2.14,6.57$ & $<0.001 *$ & 8.33 & $4.4 I, 14.9$ & $<0.00 I^{*}$ \\
\hline \multicolumn{7}{|l|}{ Ultrasound results } \\
\hline Normal & Ref & & & Ref & & \\
\hline Single anomaly & 0.60 & $0.49,0.73$ & $<0.00 I^{*}$ & 1.81 & $1.28,2.51$ & $<0.001 *$ \\
\hline Composite anomaly & 1.30 & $0.92,1.79$ & 0.114 & 2.79 & $1.84,4.15$ & $<0.001 *$ \\
\hline \multicolumn{7}{|l|}{ Maternal history } \\
\hline Normal & Ref & & & Ref & & \\
\hline Abnormal & 0.45 & $0.32,0.62$ & $<0.001 *$ & 1.06 & $0.73,1.50$ & 0.750 \\
\hline \multicolumn{7}{|c|}{ Down's screening and/or noninvasive DNA testing } \\
\hline Normal & Ref & & & Ref & & \\
\hline High risk of trisomy 13 & 6.19 & $2.79,12.3$ & $<0.001 *$ & 7.86 & $3.32,16.9$ & $<0.001 *$ \\
\hline High risk of trisomy 18 & 5.14 & $3.71,7.09$ & $<0.001 *$ & 8.68 & $5.56,13.4$ & $<0.001 *$ \\
\hline High risk of trisomy 21 & 2.14 & I.7I, $2.7 \mid$ & $<0.001 *$ & 4.07 & $2.78,5.92$ & $<0.001 *$ \\
\hline Sex chromosome abnormality & 8.91 & $6.46,12.3$ & $<0.00 I^{*}$ & 12.4 & 7.91, 19.3 & $<0.001 *$ \\
\hline Other anomaly & 1.88 & $0.83,3.70$ & 0.093 & 2.75 & $\mathrm{I} .15,5.84$ & $0.014^{*}$ \\
\hline Composite anomaly & 4.86 & $0.76,17.5$ & $0.037^{*}$ & 10.9 & $1.67,41.1$ & $0.002^{*}$ \\
\hline Neither was tested & 1.51 & $1.20,1.91$ & $<0.001 *$ & 1.05 & $0.72,1.52$ & 0.801 \\
\hline \multicolumn{7}{|l|}{ Parental chromosome } \\
\hline Both are normal & Ref & & & - & - & - \\
\hline One or both of them are abnormal & 0.81 & $0.5 \mathrm{I}, \mathrm{I} .23$ & 0.364 & - & - & - \\
\hline \multicolumn{7}{|c|}{ History of exposure to hazardous substances } \\
\hline No & Ref & & & Ref & & \\
\hline Yes & 0.39 & $0.16,0.81$ & $0.024 *$ & 0.97 & $0.38,2.04$ & 0.950 \\
\hline \multicolumn{7}{|l|}{ Twins } \\
\hline No & Ref & & & Ref & & \\
\hline Yes & 1.61 & $1.15,2.21$ & $0.004 *$ & 1.93 & I.3I, 2.80 & $<0.001 *$ \\
\hline \multicolumn{7}{|l|}{ Familial disease } \\
\hline No & Ref & & & - & - & - \\
\hline Yes & 0.35 & $0.02,1.58$ & 0.296 & - & - & - \\
\hline
\end{tabular}

Note: *Means $\mathrm{P}<0.05$.

Abbreviations: OR, odds ratio; $\mathrm{Cl}$, confidence interval; NT, nuchal translucency; NIPT, non-invasive prenatal testing; Ref, reference. 


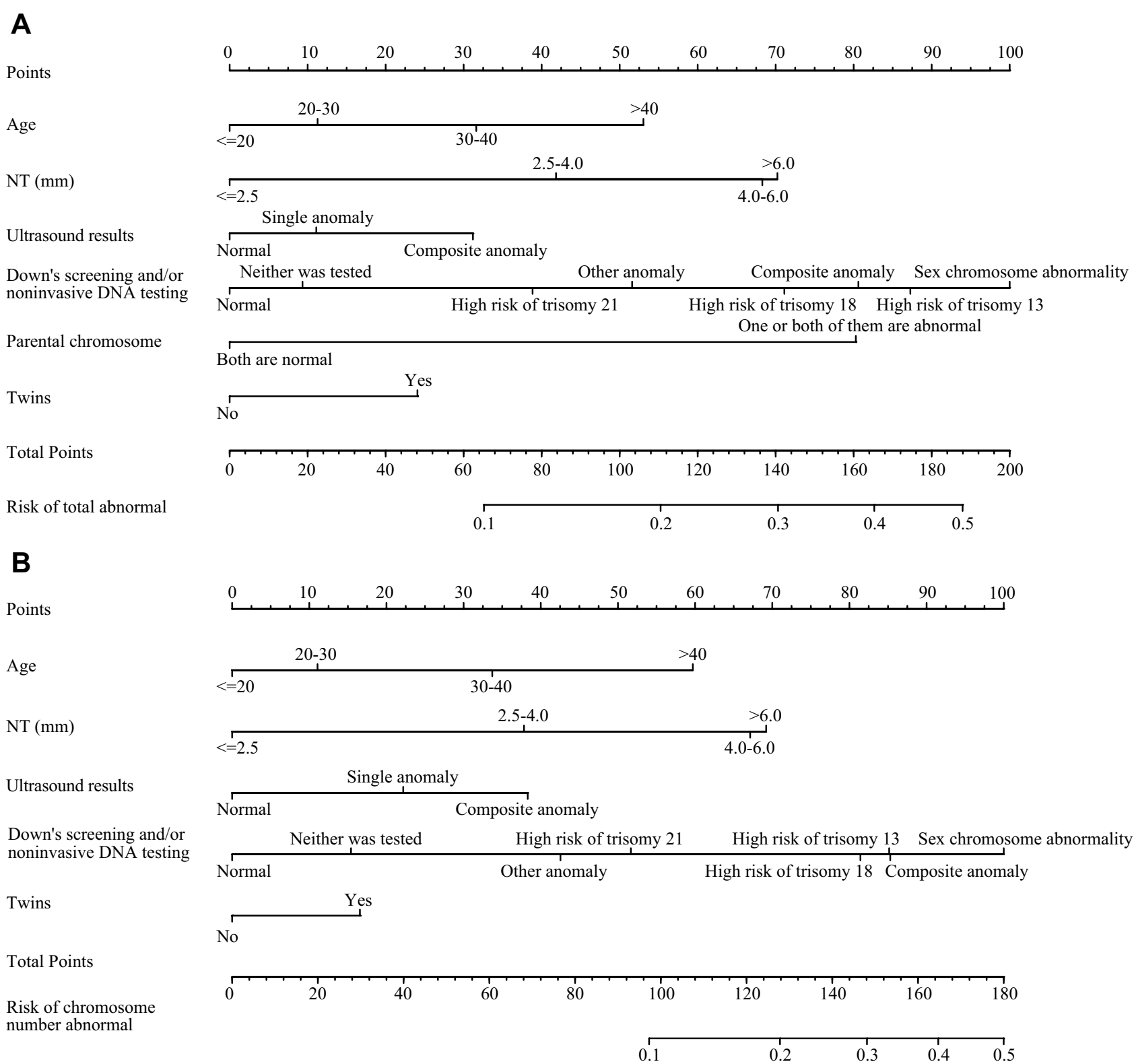

Figure I Nomograms of total abnormalities and chromosomal number abnormalities. (A) Nomograms of total abnormalities. (B) Nomograms of chromosomal number abnormalities.

abnormalities such as balanced chromosomal translocations, chromosomal microdeletions, or microduplications. The positive predictive value of NIPT was associated with the incidence of disease in the tested population. Sensitivity and specificity were similar in the low-risk population when compared to the high-risk population, but the positive predictive value was reduced. ${ }^{29,30}$ Therefore, NIPT test results must be treated with caution during prenatal genetic counseling.

Prenatal ultrasound, with its physical characteristics, can directly observe the morphology and structure of fetal tissues and organs by imaging, which is one of the non-invasive detection methods widely used in clinical practice. Studies by Karaoguz, ${ }^{31}$ Yang $^{32}$ and Tseng ${ }^{33}$ showed that the detection rates of fetal chromosomal abnormality were $5.3 \%, 6.5 \%$, and $8.9 \%$, respectively, using ultrasound for prenatal diagnosis. In conclusion, although there is a certain correlation between ultrasound and chromosomal abnormalities, its clinical value should not be overstated. ${ }^{34}$ Most of the positive ultrasonographic indicators have a good prognosis. However, NT thickening is currently recognized as the most closely associated sonographic index with trisomy 21 at $11-13^{+6}$ weeks of gestation. ${ }^{35,36}$ The $99^{\text {th }}$ percentile value of NT is $3.5 \mathrm{~mm}$, 
A

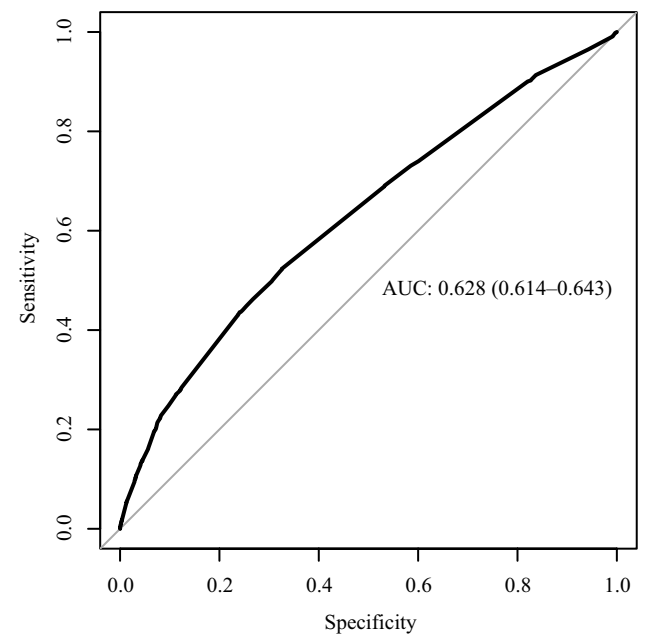

B

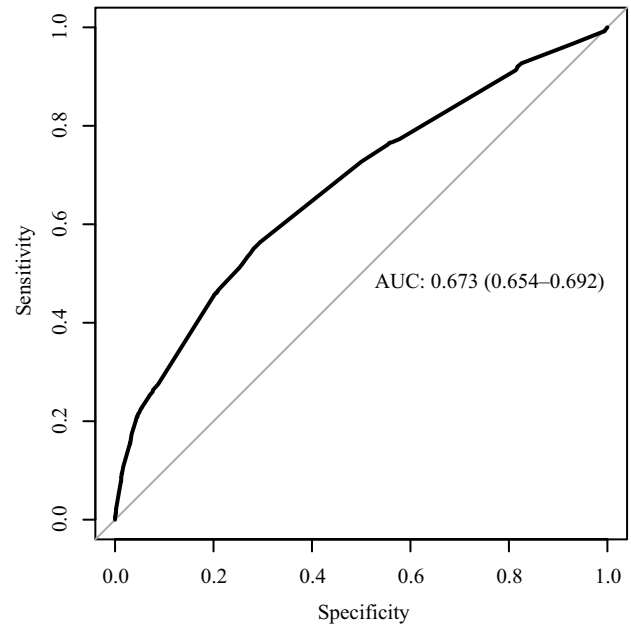

Figure 2 ROC curve of nomograms used to evaluate total abnormalities and chromosomal number abnormality. (A) Nomograms of total abnormalities. (B) Nomograms of chromosomal number abnormalities.

while the $95^{\text {th }}$ percentile value is $2.7 \mathrm{~mm} .{ }^{37}$ Some fetuses with chromosomal abnormalities of aneuploidy may also be missed with this technique. Chromosome balanced translocation is the most common chromosomal structural abnormality in the human population. Due to the different separation methods of spindle apparatus in the process of meiosis, balanced translocation carriers can form 18 types of gametes, which may lead to a very high probability of chromosomal abnormality in their offspring. Parental chromosomal examinations are very important when abnormalities are found during prenatal examinations. If the parents' chromosome karyotype analysis is normal, fetal chromosomal abnormalities may be due to a new mutation, that is, chromosomal microdeletion, duplication, or gene mutation, which can further improve fetal gene$\mathrm{CNV}$ (copy number variation) or whole-genome sequencing. Alternatively, a fetus with a normal clinical phenotype may be recommended to be retained even if no abnormality is found on prenatal examination, and one of the parents is a carrier of chromosomal abnormality. Therefore, counseling or prediction in prenatal diagnosis is needed to reduce unnecessary invasive tests.

Previous studies have reported that the indications for prenatal diagnosis also need to consider the adverse pregnancy history, family genetic disorder history, and toxicological exposure history of pregnant women. However, our model analyzed the influence of these factors on the results of fetal karyotype analysis and found that they had no effect on the results. Fetal chromosomal karyotype abnormalities (abnormal numbers) are mainly related to clinical factors such as maternal age, NT, Oscar's testing, NIPT, ultrasound anomalies and parental chromosomal factors.

Fetal chromosomal disorders include abnormal number, abnormal structure, and polymorphism of chromosomes. Among them, chromosomal aneuploidy caused by abnormal chromosome number is the most common chromosomal disorder in clinical practice, accounting for $30 \%-50 \%$ of all pregnancies with chromosomal abnormalities. $^{38}$ In addition, chromosomal microdeletions and microduplications are chromosomal disorders caused by submicroscopic chromosomal deletions or duplications that lead to normal gene imbalance. ${ }^{39}$ These constitute another important genetic factors of fetal birth defects, which account for about $15 \%$ of all inherited diseases. ${ }^{40}$ However, since fetal chromosome karyotype analysis can only identify chromosomal variation greater than $5 \mathrm{MBP}$, the positive results of $\mathrm{CNV}$ were not predicted in this study. In addition, chromosomal polymorphism is not pathogenic, so in our study, only the TA and CNA identified as pathogenic were discussed. For structural abnormalities and polymorphism, we also completed the prediction model, but the results were not consistent with the clinical practice, so they are presented in the supporting document.

Our nomogram was developed on the basis of the six years of clinical data from the hospital, with a large sample size to ensure the reliability and stability of the results. Nomogram curve analysis and internal verification show that this method has good discriminant and calibration 
A

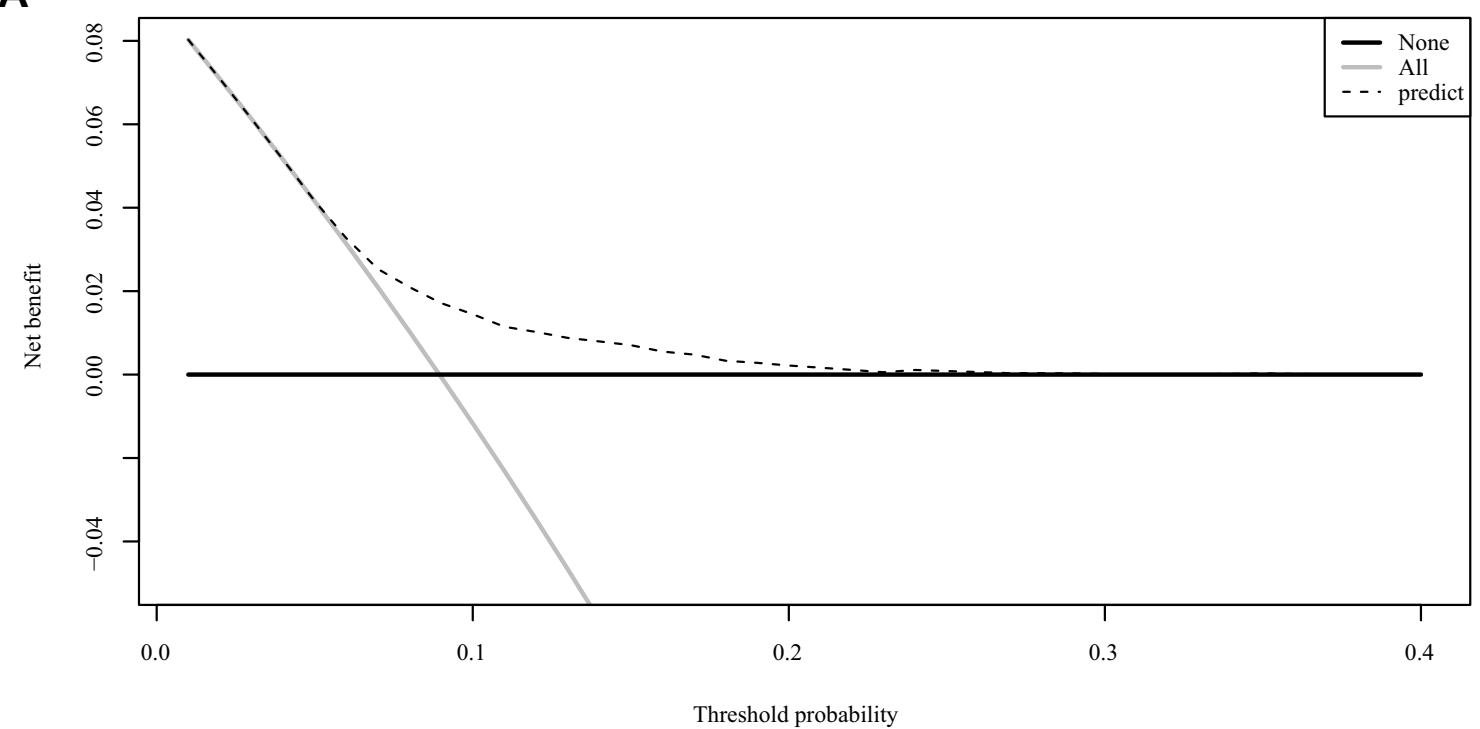

B

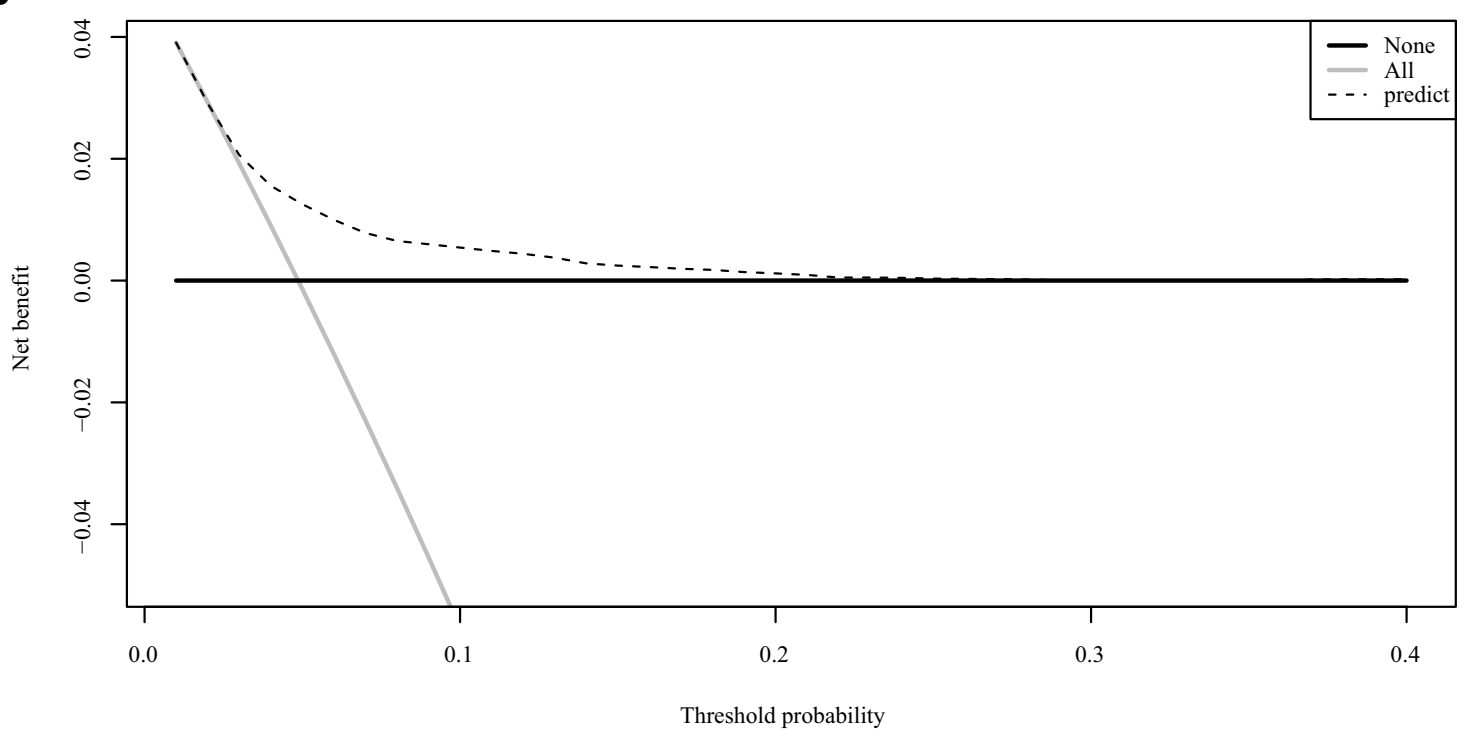

Figure 3 Decision curve analysis (DCA) of both nomograms. (A) Nomograms of total abnormalities. (B) Nomograms of chromosomal number abnormalities.

capabilities. Nomograms can be used to effectively screen high-risk patients with fetal chromosomal abnormalities and to provide reference for patients to receive amniocentesis. DCA is very useful in determining whether modelbased clinical decisions are effective, while traditional ROC curve analysis is a statistical abstraction method and cannot provide information about clinical value directly. ${ }^{41}$ Clinical practicality is an important indicator to judge whether the prediction model can be used in clinical activities and whether patients can benefit from it; however, few studies have used this new method to evaluate the net benefits of predictive models, and even fewer have applied it to predictive models for prenatal diagnosis. C Mazouni ${ }^{42}$ used a nomogram to assess the risk of macrosomia based on parity, ethnicity, body mass index, and fetal weight to estimate macrosomia, which had good discrimination and calibration before and after bootstrapping. Our team has also used DCA curves to evaluate the clinical practicality of our model. ${ }^{43,44}$ In this model, the nomogram's net benefit was better than that in allpatient-negative-risk or all-patient-positive-risk scenarios at a threshold probability between $0 \%$ and $60 \%$.

Although we developed the first nomogram to construct amniocentesis results based on extensive clinical data, our current work has some limitations. First, our study did not focus on the entire population, but only on the population that 
A

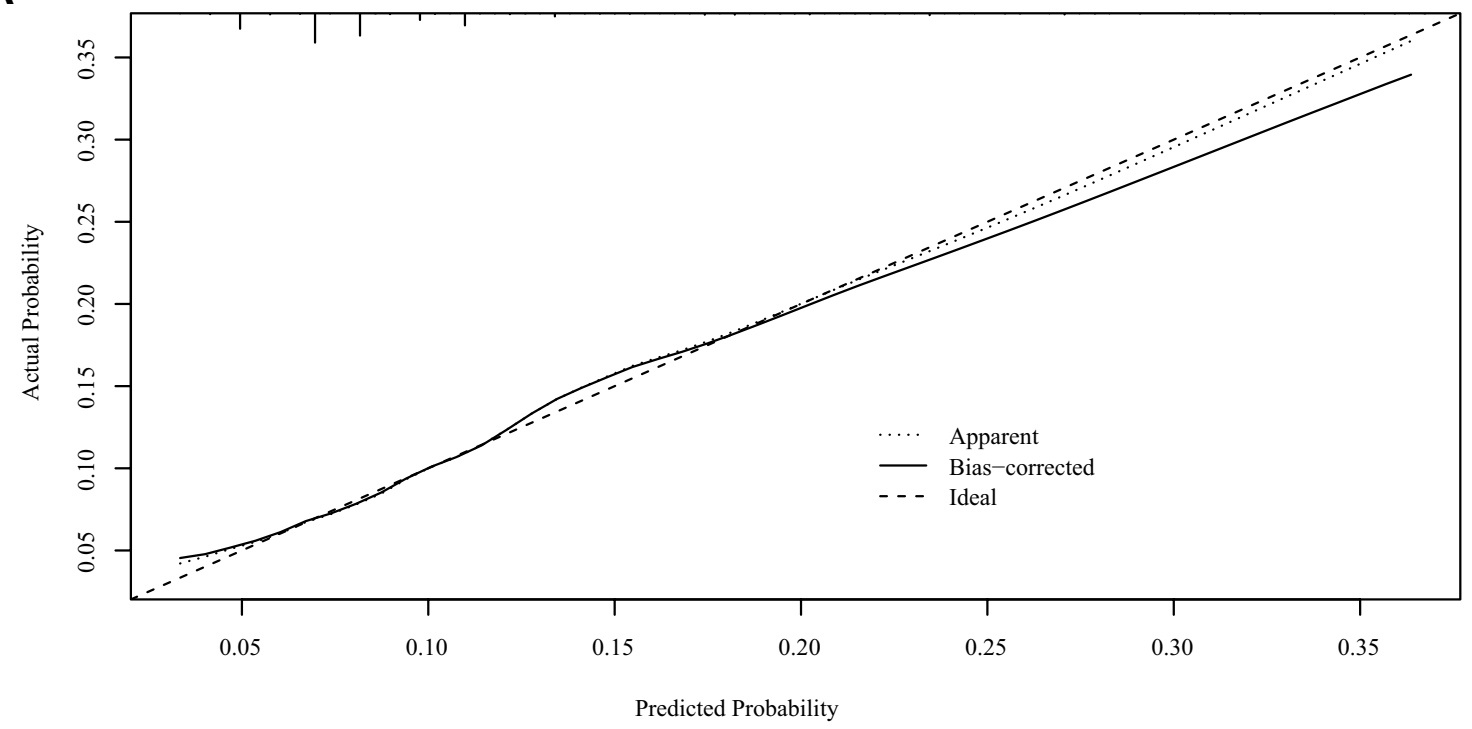

B

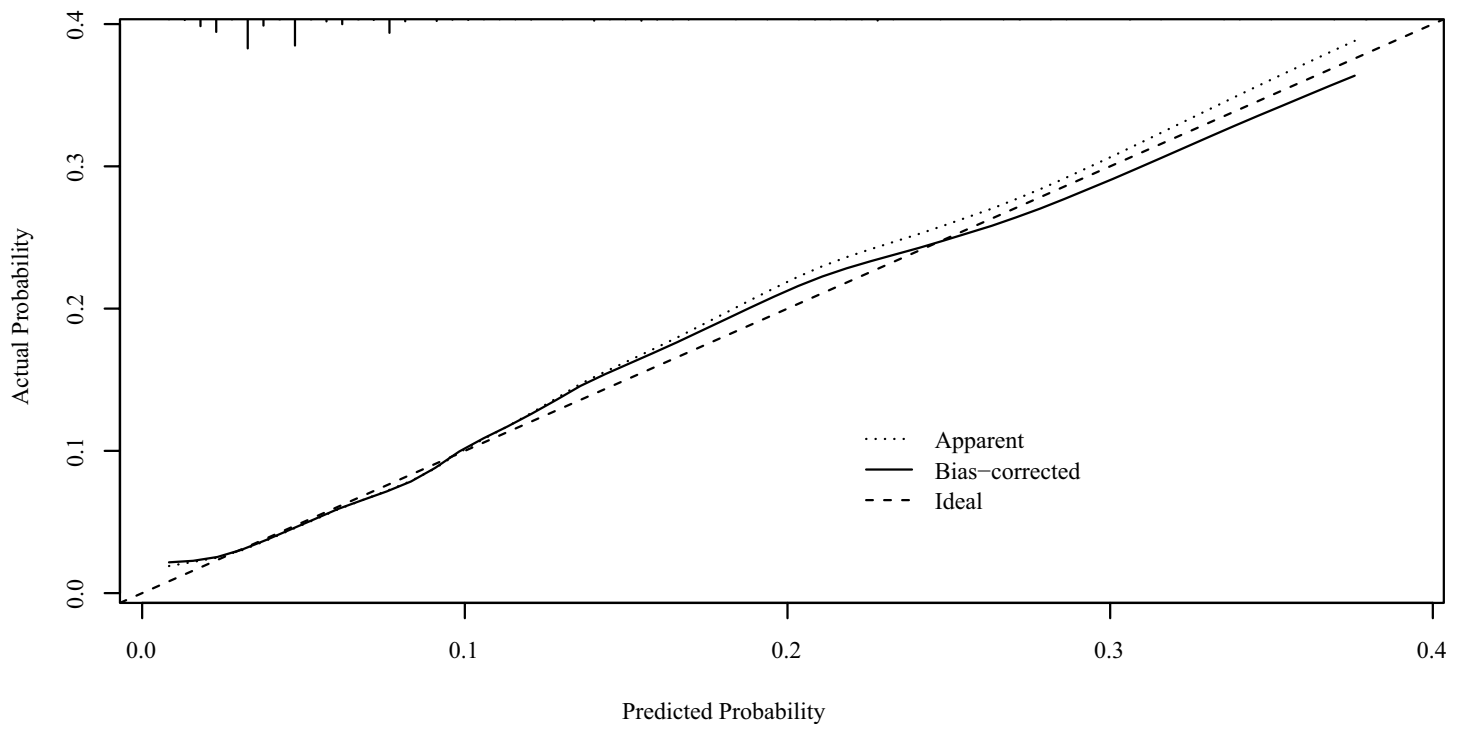

Figure 4 Calibration curves of both nomograms. (A) Nomograms of total abnormalities. (B) Nomograms of chromosomal number abnormalities.

underwent amniocentesis for a variety of abnormal reasons, and there may be a selection bias due to this. Our study also has the limitations of a retrospective review for data bias. In addition, the model has not been externally verified to ensure the generality of our model. Future research can be combined with data from other centers to make better predictions.

Table 4 C-Statistic and Brier Score of Nomograms

\begin{tabular}{|l|c|c|c|c|}
\hline \multirow{2}{*}{ Characteristics } & \multicolumn{2}{|c|}{ C-Statistic } & \multicolumn{2}{c|}{ Brier Score } \\
\cline { 2 - 5 } & $\begin{array}{c}\text { Training } \\
\text { Cohort }\end{array}$ & $\begin{array}{c}\text { After internal } \\
\text { Verification }\end{array}$ & $\begin{array}{c}\text { Training } \\
\text { Cohort }\end{array}$ & $\begin{array}{c}\text { After Internal } \\
\text { Verification }\end{array}$ \\
\hline Total abnormalities & 0.6339 & 0.6287 & 0.0791 & 0.0794 \\
Chromosome number & 0.6831 & 0.6781 & 0.0443 & 0.0444 \\
abnormality & & & & \\
\hline
\end{tabular}




\section{Conclusions}

The nomogram constructed in this study is a good predictor of total amniocentesis abnormalities and chromosomal number abnormalities. This study is retrospective and more prospective or multicenter studies should be performed before its use in clinical practice for high-risk patient of chromosomal abnormalities.

\section{Capsule}

The nomogram is a good predictor of total amniocentesis abnormalities and chromosomal number abnormalities and can be used in clinical practice for high-risk patients.

\section{Details of Ethics Approval}

All procedures were approved by Ethics Committee of Shengjing Hospital of China Medical University (reference number 2020PS284K, approved 01/04/2020).

\section{Acknowledgments}

We would like to express our gratitude to all those who helped us during the writing of this manuscript. Thanks to Prenatal Diagnostic Center of Liaoning Province for providing data resources. Thanks to all the peer reviewers for their opinions and suggestions.

\section{Funding}

This study was supported by internal funding of Shengjing Hospital, China Medical University (SJ-M0133).

\section{Disclosure}

The authors have no conflicts of interest to declare.

\section{References}

1. Blue NR, Page JM, Silver RM. Genetic abnormalities and pregnancy loss. Semin Perinatol. 2019;43(2):66-73. doi:10.1053/j. semperi.2018.12.002

2. Linden MG, Bender BG, Robinson A. Genetic counseling for sex chromosome abnormalities. Am J Med Genet. 2002;110(1):3-10. doi:10.1002/ajmg.10391

3. Ou S, Ou H, Tang B, Chen SK, Xu YQ, Zheng CG. Analysis of chromosomal abnormalities and a report of eight new karyotypes among children in genetic counseling. Zhongguo Dang Dai Er Ke Za Zhi. 2014;16(7):725-728.

4. Jackson LG, Zachary JM, Fowler SE, et al. A randomized comparison of transcervical and transabdominal chorionic-villus sampling. The U.S. National Institute of Child Health and Human Development Chorionic-Villus Sampling and Amniocentesis Study Group. $N$ Engl J Med. 1992;327(9):594-598. doi:10.1056/ NEJM199208273270903

5. Jummaat F, Ahmad S, Mohamed Ismail NA. 5-Year review on amniocentesis and its maternal fetal complications. Horm Mol Biol Clin Investig. 2019;40(2). doi:10.1515/hmbci-2019-0006
6. Seeds JW. Diagnostic mid trimester amniocentesis: how safe? Am $J \quad$ Obstet Gynecol. 2004;191(2):607-615. doi:10.1016/j. ajog.2004.05.078

7. Han Z, Zhang Y, Bai X, Yin Y, Xu C, Hou H. Mother-to-child transmission of hepatitis $\mathrm{B}$ virus after amniocentesis: a retrospective matched cohort study. Prenat Diagn. 2019;39(6):431-440. doi: $10.1002 /$ pd. 5452

8. Mandelbrot L, Jasseron C, Ekoukou D, et al. Amniocentesis and mother-to-child human immunodeficiency virus transmission in the Agence Nationale de Recherches sur le SIDA et les Hépatites Virales French Perinatal Cohort. Am J Obstet Gynecol. 2009;200(2):160.e19. doi:10.1016/j.ajog.2008.08.049

9. Carlson LM, Vora NL. Prenatal Diagnosis: screening and Diagnostic Tools. Obstet Gynecol Clin North Am. 2017;44(2):245-256. doi:10.1016/j.ogc.2017.02.004

10. Reid R, Sepulveda W, Kyle PM, Davies G. Amniotic fluid culture failure: clinical significance and association with aneuploidy. Obstet Gynecol. 1996;87(4):588-592. doi:10.1016/0029-7844(95) 00479-3

11. Ghi T, Sotiriadis A, Calda P, et al. ISUOG Practice Guidelines: invasive procedures for prenatal diagnosis. Ultrasound Obstetrics Gynecol. 2016;48(2):256-268. doi:10.1002/uog.15945

12. ACOG Practice Bulletin No. 27: Clinical Management Guidelines for Obstetrician-Gynecologists. Prenatal diagnosis of fetal chromosomal abnormalities. Obstet Gynecol. 2001;97(5Pt 1):suppl 1-12.

13. Amniocentesis (Consent Advice No. 6); 2006. Available from: https://www.rcog.org.uk/en/guidelines-research-services/guidelines/ consent-advice-6/. Accessed October 18, 2021.

14. Xie G, Wang R, Shang L, et al. Calculating the overall survival probability in patients with cervical cancer: a nomogram and decision curve analysis-based study. BMC Cancer. 2020;20(1):833. doi:10.1186/s12885-020-07349-4

15. Zhu L, Sun X, Bai W. Nomograms for Predicting Cancer-Specific and Overall Survival Among Patients With Endometrial Carcinoma: a SEER Based Study. Front Oncol. 2020;10(10):269. doi:10.3389/ fonc. 2020.00269

16. Lisa Shaffer -JM-J, Schmid M. ISCN 2013: An International System for Human Cytogenetic Nomenclature (2013). Karger; 2013.

17. Janssens A, Martens FK. Reflection on modern methods: revisiting the area under the ROC Curve. Int $J$ Epidemiol. 2020;1 (49):1397-1403. doi:10.1093/ije/dyz274

18. Pencina MJ, D'Agostino RB. Overall $\mathrm{C}$ as a measure of discrimination in survival analysis: model specific population value and confidence interval estimation. Stat Med. 2004;23(13):2109-2123. doi: $10.1002 / \operatorname{sim} .1802$

19. Roulston M. Performance targets and the brier score. Meteorol Applications. 2007;14(2):185-194. doi:10.1002/met.21

20. Van Calster B, Wynants L, Verbeek JFM, et al. Reporting and Interpreting Decision Curve Analysis: a Guide for Investigators. Eur Urol. 2018;74(6):796-804. doi:10.1016/j.eururo.2018.08.038

21. Levy B, Stosic M. Traditional Prenatal Diagnosis: past to Present. Methods Mol Biol. 2019:3-22.

22. Ferrari M, Carrera P, Lampasona V, Galbiati S. New trend in non-invasive prenatal diagnosis. Clin Chim Acta. 2015;451(Pt A):9-13. doi:10.1016/j.cca.2014.12.026

23. Pergament E. First-trimester genetic counseling: perspectives and considerations. Clin Lab Med. 2010;30(3):557-563. doi:10.1016/j. cll.2010.05.004

24. Yu B, Li H, Chen YP, et al. Clinical evaluation of NIPS for women at advanced maternal age: a multicenter retrospective study. $j$ MaternalFetal Neonatal Med. 2019;32(24):4080-4085. doi:10.1080/ 14767058.2018.1481385

25. Nakata N, Wang Y, Bhatt S. Trends in prenatal screening and diagnostic testing among women referred for advanced maternal age. Prenat Diagn. 2010;30(3):198-206. doi:10.1002/pd.2434 
26. Godino L, Pompilii E, D'Anna F, et al. Attitudes of women of advanced maternal age undergoing invasive prenatal diagnosis and the impact of genetic counselling. Eur j Human Genetics. 2016;24 (3):331-337. doi:10.1038/ejhg.2015.116

27. Juvet LK, Ormstad SS, Stoinska-Schneider A, et al. NIPH Systematic Reviews: Executive Summaries. Non-Invasive Prenatal Test (NIPT) for Identification of Trisomy 21, 18 and 13. Oslo, Norway: Knowledge Centre for the Health Services at The Norwegian Institute of Public Health (NIPH) Copyright (C) 2016 by The Norwegian Institute of Public Health (NIPH); 2016.

28. Gil MM, Accurti V, Santacruz B, Plana MN, Nicolaides KH. Analysis of cell-free DNA in maternal blood in screening for aneuploidies: updated meta-analysis. Ultrasound Obstetrics Gynecol. 2017;50(3):302-314. doi:10.1002/uog.17484

29. Benn P, Borrell A, Chiu RW, et al. Position statement from the Chromosome Abnormality Screening Committee on behalf of the Board of the International Society for Prenatal Diagnosis. Prenat Diagn. 2015;35(8):725-734. doi:10.1002/pd.4608

30. The American College of Obstetricians and Gynecologists SfM-FM. Committee Opinion Summary No. 640: cell-Free DNA Screening For Fetal Aneuploidy. Obstet Gynecol. 2015;126(3):691-692. doi:10.1097/01.AOG.0000471171.86798.ac

31. Karaoguz MY, Bal F, Yakut T, et al. Cytogenetic results of amniocentesis materials: incidence of abnormal karyotypes in the Turkish collaborative study. Genetic Counseling. 2006;17(2):219-230.

32. Tseng JJ, Chou MM, Lo FC, Lai HY, Chen MH, Ho ES. Detection of chromosome aberrations in the second trimester using genetic amniocentesis: experience during 1995-2004. Taiwan J Obstet Gynecol. 2006;45(1):39-41. doi:10.1016/S1028-4559(09)60188-1

33. Yang YH, Ju KS, Kim SB, et al. The Korean collaborative study on 11,000 prenatal genetic amniocentesis. Yonsei Med J. 1999;40 (5):460-466. doi:10.3349/ymj.1999.40.5.460

34. Gomez KJ, Copel JA. Ultrasound screening for fetal structural anomalies. Curr Opin Obstet Gynecol. 1993;5(2):204-210. doi:10.1097/00001703-199304000-00006
35. Spaggiari E, Czerkiewicz I, Sault C, et al. Impact of Including or Removing Nuchal Translucency Measurement on the Detection and False-Positive Rates of First-Trimester Down Syndrome Screening. Fetal Diagn Ther. 2016;40(3):214-218. doi:10.1159/000442198

36. Nicolaides KH. Screening for fetal aneuploidies at 11 to 13 weeks. Prenat Diagn. 2011;31(1):7-15. doi:10.1002/pd.2637

37. Manotaya S, Zitzler J, Li X, et al. Effect of ethnicity on first trimester biomarkers for combined trisomy 21 screening: results from a multicenter study in six Asian countries. Prenat Diagn. 2015;35 (8):735-740. doi:10.1002/pd.4602

38. Wieacker P, Steinhard J. The prenatal diagnosis of genetic diseases. Dtsch Arztebl Int. 2010;107(48):857-862.

39. Wu XL, Li R, Fu F, et al. Chromosome microarray analysis in the investigation of children with congenital heart disease. BMC Pediatr. 2017;17(1):117. doi:10.1186/s12887-017-0863-3

40. Vissers LE, Veltman JA, van Kessel AG, Brunner HG. Identification of disease genes by whole genome CGH arrays. Hum Mol Genet. 2005;14:R215-23. doi:10.1093/hmg/ddi268

41. Vickers AJ, Van Calster B, Steyerberg EW. Net benefit approaches to the evaluation of prediction models, molecular markers, and diagnostic tests. BMJ. 2016;352:i6. doi:10.1136/bmj.i6

42. Mazouni C, Rouzier R, Ledu R, Heckenroth H, Guidicelli B, Gamerre M. Development and internal validation of a nomogram to predict macrosomia. Ultrasound Obstetrics Gynecol. 2007;29 (5):544-549. doi:10.1002/uog.3999

43. Chen B, Zhang L, Wang D, et al. Nomogram to predict postpartum hemorrhage in cesarean delivery for women with scarred uterus: a retrospective cohort study in China. J Obstet Gynaecol Res. 2020;46(9):1772-1782. doi:10.1111/jog.14354

44. Zhang L, Li H, Li J, et al. Prediction of iatrogenic preterm birth in patients with scarred uterus: a retrospective cohort study in Northeast China. BMC Pregnancy Childbirth. 2020;20(1):490. doi:10.1186/ s12884-020-03165-7
Risk Management and Healthcare Policy

\section{Publish your work in this journal}

Risk Management and Healthcare Policy is an international, peerreviewed, open access journal focusing on all aspects of public health, policy, and preventative measures to promote good health and improve morbidity and mortality in the population. The journal welcomes submitted papers covering original research, basic science, clinical \& epidemiological studies, reviews and evaluations, guidelines, expert opinion and commentary, case reports and extended reports. The manuscript management system is completely online and includes a very quick and fair peer-review system, which is all easy to use. Visit http://www.dovepress.com/testimonials.php to read real quotes from published authors. 